\title{
Tracheal Fistula, CTCAE
}

National Cancer Institute

\section{Source}

National Cancer Institute. Tracheal Fistula, CT CAE. NCI Thesaurus. Code C58205.

A disorder characterized by an abnormal communication between the trachea and another organ or anatomic site. 